\title{
Chewing at the ends
}

\section{DOI:}

$10.1038 / \mathrm{nrm} 2301$
DNA double-strand breaks (DSBs) that occur in the $S$ and $G 2$ phases of the cell cycle are mainly repaired by homologous recombination (HR). HR is initiated by resection of the DNA strands surrounding a DSB, resulting in single-stranded DNA (ssDNA) that is bound by replication protein A (RPA). Consequently, strand invasion (an early step of HR) and recruitment of ataxia-telangiectasia and RAD3-related kinase (ATR; a kinase that mediates cell cycle checkpoint activation) establish the DNA-damage response. Stephen Jackson and colleagues now elaborate on the mechanism and control of DSB resection, a crucial step in the activation of specific DNA-damageresponse pathways.

The role of human CtIP (also known as $\underline{\mathrm{RBBP} 8}$ ) in DNA-damage signalling has remained unclear, despite previous reports that it interacts with the DNAdamage-response proteins BRCA1 and RAD50. Jackson and colleagues showed that depletion of CtIP, mediated by small interfering $\mathrm{RNA}$, in U2OS cells results in hypersensitivity to camptothecin and etoposide - drugs that induce DSBs in $S$ phase. Moreover, following drug treatment, CtIP was required for the effective phosphorylation of $\mathrm{CHK} 1$, a substrate of ATR that mediates cell cycle checkpoint activation. Analysis of CtIP recruitment to laser-induced tracks of DSBs showed that CtIP redistributes to the tracks in cells that were expressing cyclin $\mathrm{A}$, which is indicative of $S$ and G2 phases. Consistently, CtIP-depleted cells showed a reduced ability to recruit ATR to laser tracks, and ssDNA formation and RPA recruitment was significantly reduced after camptothecin treatment, indicating that DSB resection is defective in CtIP-depleted cells. Together, this implies that CtIP is required to promote ATR-dependent signalling in response to DSBs that occur in S phase and therefore require resection.

Efficient DSB resection was previously shown to require the MRE11-RAD50-NBS1 (MRN) complex, and Jackson and colleagues showed that CtIP directly interacts with this complex. Moreover, CtIP cooperated with MRE11-RAD50 in vitro to exhibit endonuclease activity, and depletion of either CtIP or MRE11 decreased the frequency of HR. Simultaneous depletion of both MRE11 and CtIP did not additively reduce the frequency of $\mathrm{HR}$, indicating that they function together to promote HR.

DNA-damage-response signalling is well conserved from humans to yeast, and Jackson and colleagues showed that CtIP has homology to Saccharomyces cerevisiae Sae2, which cooperates with the homologous yeast MRN complex to mediate DSB resection and checkpoint activation.
In a separate paper, Paul Russell and colleagues identified the homologue of CtIP in Schizosaccharomyces pombe, called Ctp1, which is periodically expressed in S and G2 phases. They showed that $c t p 1 \Delta$ cells were hypersensitive to ionizing radiation and camptothecin, and that Ctp1 is required for the repair of programmed DSBs that occur in meiosis and for HR repair. Consistently, they also showed that Ctp1 was epistatic with members of the homologous MRN complex and that these proteins function together to mediate strand resection surrounding DSBs that are induced during $\mathrm{S}$ and G2 phase.

Therefore, CtIP and its yeast homologues appear to regulate strand resection and, hence, the DNA-damage response according to the phases of the cell cycle. Indeed, CtIP is hyperphosphorylated in response to DSBs, and numerous consensus sites for ATR (and related kinases) and cyclin-dependent kinases (CDKs) are evident in the CtIP primary sequence. Interestingly, context-specific phosphorylation of CtIP might determine which DNA-damage-response pathways are activated.

Gemma K. Alderton

ORIGINAL RESEARCH PAPERS Sartori, A. A. et al. Human CtIP promotes DNA end resection. Nature 28 Oct 2007 (doi:10.1038/nature06337) | Limbo, O. et al. Ctp1 is a cell-cycle-regulated protein that functions with Mre11 complex to control double-strand break repair by homologous recombination. Mol. Cell 28 134-146 (2007) 